\title{
Backward Aluthge iterates of a hyponormal operator and scalar extensions
}

\author{
by \\ C. Benhida (Lille) and E. H. Zerouali (Rabat)
}

\begin{abstract}
Let $R$ and $S$ be two operators on a Hilbert space. We discuss the link between the subscalarity of $R S$ and $S R$. As an application, we show that backward Aluthge iterates of hyponormal operators and $p$-quasihyponormal operators are subscalar.
\end{abstract}

1. Introduction. Let $\mathcal{H}$ be a Hilbert space and $\mathcal{L}(\mathcal{H})$ be the algebra of bounded linear operators on $\mathcal{H}$. We write $\sigma(T)$ for the spectrum of $T$.

In [14], M. Putinar showed that a hyponormal operator has a scalar extension which means that it is similar to the restriction to an invariant subspace of a (generalized) scalar operator (in the sense of Colojoară-Foiaş). This was extended to $w$-hyponormal operators by E. Ko [10].

A bounded linear operator $S$ on $\mathcal{H}$ is called scalar of order $m$ if it has a spectral distribution of order $m$, i.e., if there is a continuous unital morphism of topological algebras

$$
\Phi: C_{0}^{m}(\mathbb{C}) \rightarrow \mathcal{L}(\mathcal{H})
$$

such that $\Phi(z)=S$, where as usual $z$ stands for the identical function on $\mathbb{C}$ and $C_{0}^{m}(\mathbb{C})$ for the space of compactly supported functions on $\mathbb{C}$, continuously differentiable of order $m, 0 \leq m \leq \infty$. We mention that here we will identify any function with its values in a neighborhood of the spectrum of $S$. An operator is subscalar of order $m$ if it is a restriction of a scalar operator of order $m$. It is known that subscalarity of order $\infty$ is equivalent to property $(\beta)_{\epsilon}[7$, Proposition 3.3].

A Banach space operator $A \in \mathcal{L}(\mathcal{X})$ has property $(\beta)_{\epsilon}$ if, for each open subset $\mathcal{U}$ of the complex plane $\mathbb{C}$, the operator

$$
A_{z}: \mathcal{E}(\mathcal{U}, \mathcal{X}) \rightarrow \mathcal{E}(\mathcal{U}, \mathcal{X})
$$

defined by $A_{z}(f)=(A-z) f$ from the Fréchet space $\mathcal{E}(\mathcal{U}, \mathcal{X})$ of $\mathcal{X}$-valued $C^{\infty}$-functions into itself is one-to-one and has closed range. 
Property $(\beta)_{\epsilon}$ implies (Bishop's) property $(\beta)$, where an operator $A \in$ $\mathcal{L}(\mathcal{X})$ is said to have property $(\beta)$ if, for each open subset $\mathcal{U}$ of $\mathbb{C}$, the operator

$$
A_{z}: \mathcal{O}(\mathcal{U}, \mathcal{X}) \rightarrow \mathcal{O}(\mathcal{U}, \mathcal{X})
$$

from the Fréchet space $\mathcal{O}(\mathcal{U}, \mathcal{X})$ of $\mathcal{X}$-valued analytic functions on $\mathcal{U}$ into itself is one-to-one and has closed range. Properties $(\beta)_{\epsilon}$ and $(\beta)$ play an important role in local spectral theory and related topics. For further information, see $[1,6,7]$ and [13].

Let $\mathbf{D}$ be a bounded open disc in $\mathbb{C}$ and $d \mu(z)$ the planar Lebesgue measure. We shall denote by $L^{2}(\mathbf{D}, \mathcal{H})$ the Hilbert space of measurable functions $f: \mathbf{D} \rightarrow \mathcal{H}$ such that

$$
\|f\|_{2, \mathbf{D}}=\left(\int_{\mathbf{D}}\|f(z)\|^{2} d \mu(z)\right)^{1 / 2}<\infty .
$$

The space of functions $f \in L^{2}(\mathbf{D}, \mathcal{H})$ which are analytic in $\mathbf{D}$ is defined by

$$
A^{2}(\mathbf{D}, \mathcal{H})=L^{2}(\mathbf{D}, \mathcal{H}) \cap \mathcal{O}(\mathbf{D}, \mathcal{H})
$$

where $\mathcal{O}(\mathbf{D}, \mathcal{H})$ denotes the Fréchet space of $\mathcal{H}$-valued analytic functions on $\mathbf{D}$ with uniform topology. $A^{2}(\mathbf{D}, \mathcal{H})$ is called the Bergman space for $\mathbf{D}$.

Let us define a Sobolev type space $W^{m}(\mathbf{D}, \mathcal{H})$, for $m \in \mathbb{N}$, to be the space of those functions $f \in L^{2}(\mathbf{D}, \mathcal{H})$ whose derivatives $\bar{\partial} f, \ldots, \bar{\partial}^{m} f$ in the sense of distributions still belong to $L^{2}(\mathbf{D}, \mathcal{H})$. Endowed with the norm

$$
\|f\|_{W^{m}}^{2}=\sum_{i=0}^{m}\left\|\bar{\partial}^{i} f\right\|_{2, \mathbf{D}}^{2},
$$

$W^{m}(\mathbf{D}, \mathcal{H})$ becomes a Hilbert space continuously embedded in $L^{2}(\mathbf{D}, \mathcal{H})$. Now, for $f \in C_{0}^{m}(\mathbb{C})$, let $M_{f}$ denote the operator on $W^{m}(\mathbf{D}, \mathcal{H})$ given by multiplication by $f$. It has a spectral distribution of order $m$, defined by the functional calculus

$$
\Phi_{M}: C_{0}^{m}(\mathbb{C}) \rightarrow \mathcal{L}\left(W^{m}(\mathbf{D}, \mathcal{H})\right), \quad \Phi_{M}(f)=M_{f} .
$$

Thus, $M_{z}$ is a scalar operator of order $m$.

We proved in [4] that for two given operators $R$ and $S$, the operators $R S$ and $S R$ share the same local spectral properties (SVEP, $(\beta),(\beta)_{\epsilon}$, etc.) and almost all their global spectral properties.

In this paper, we consider intermediate properties between SVEP and property $(\beta)_{\epsilon}$ involving Sobolev spaces. We then investigate the behavior of $R S$ and $S R$ with regard to these properties.

As an application, we give various results on Aluthge transforms and backward Aluthge iterates of hyponormal operators and $p$-quasihyponormal operators. 
Notice in passing that such an application provides a simple approach to obtaining subscalarity of many classes of operators. Similar results are given in $[9,11,12]$. Proofs in the previous papers rely heavily on some $L^{2}$-version of the following lemma due to Xia, which can be found for example in [9].

Lemma 1.1 ([16, Lemma 2.1]). Let $T=U|T| \in \mathcal{L}(\mathcal{H})$ be the polar decomposition of $T, Q=|T|-|T|_{l}, z=\varrho e^{i \theta}, \varrho>0$, and $\left|e^{i \theta}\right|=1$ where $|T|=\left(T^{*} T\right)^{1 / 2}$ and $|T|_{l}=\left(T T^{*}\right)^{1 / 2}$. Then for all $f \in \mathcal{H}$,

$$
\|(T-z) f\|^{2}=\|(|T|-\varrho) f\|^{2}+\varrho\left\||T|^{1 / 2}\left(U-e^{i \theta}\right)^{*} f\right\|^{2}+\varrho\langle Q f, f\rangle .
$$

It is however not clear for us how such an $L^{2}$-version could be applied.

2. Subscalarity of $R S$ and $S R$. Using the Cauchy-Pompeiu formula, M. Putinar proved in [14] the lemma below for $i=0$, from which one may deduce easily the following form:

Lemma 2.1. For every bounded disc $\mathbf{D}$ in $\mathbb{C}$ there is a constant $C_{\mathbf{D}}$ such that for all $T \in B(\mathcal{H})$ and $f \in W^{m}(\mathbf{D}, \mathcal{H})$ we have

$$
\left\|(I-P) \bar{\partial}^{i} f\right\|_{2, \mathbf{D}} \leq C_{\mathbf{D}}\left[\left\|(T-z)^{*} \bar{\partial}^{i+1} f\right\|_{2, \mathbf{D}}+\left\|(T-z)^{*} \bar{\partial}^{i+2} f\right\|_{2, \mathbf{D}}\right]
$$

for $i=0,1, \ldots, m-2$, where $P$ denotes the orthogonal projection of $L^{2}(\mathbf{D}, \mathcal{H})$ onto $A^{2}(\mathbf{D}, \mathcal{H})$

We shall also use the following well known fact (see [14]):

Lemma 2.2. Let $U$ and $V$ be bounded connected open sets in $\mathbb{C}$. If $V$ is relatively compact in $U$, then there is a constant $c>0$ such that $\|f\|_{\infty, V} \leq$ $c\|f\|_{2, U}$ for all $f \in A^{2}(U, \mathcal{H})$.

In [4], we proved that for two given operators $R$ and $S$, the operators $R S$ and $S R$ have the same local spectral properties, in particular, the singlevalued extension property (SVEP). The following result shows that we have an analogue of the SVEP for Sobolev spaces.

Proposition 2.3. Let $R$ and $S$ be two operators on $\mathcal{H}$ and let $\mathbf{D}$ be a bounded disc containing $\sigma(R S) \cup\{0\}$. Then $R S-\lambda: W^{2}(\mathbf{D}, \mathcal{H}) \rightarrow W^{2}(\mathbf{D}, \mathcal{H})$ is one-to-one if and only if $S R-\lambda: W^{2}(\mathbf{D}, \mathcal{H}) \rightarrow W^{2}(\mathbf{D}, \mathcal{H})$ is one-to-one.

Proof. It is clear that by symmetry, it is enough to prove the statement in one direction. Assume that $R S-\lambda$ is one-to-one. If $f \in W^{2}(\mathbf{D}, \mathcal{H})$ is such that $(S R-\lambda) f=0$, then $(R S-\lambda) R f=0$. By hypothesis, $R f=0$. Thus $\lambda f=0$. Consequently, $\lambda \bar{\partial}^{i} f=0$ for $i=1,2$. Using Lemma 2.1 with $T=0$, we get $f=P f$. Then $\lambda f=\lambda P f=0$. But, from [5, Corollary 10.7], there exists a constant $c>0$ such that

$$
c\|P f\|_{2, \mathbf{D}} \geq\|\lambda P f\|_{2, \mathbf{D}} .
$$

Therefore $f=P f=0$. 
Let us introduce the following definition.

Definition 2.4. Let $A$ be an operator on $\mathcal{H}$, and $m$ and $p$ two integers such that $0 \leq p<m$. We shall say that $A$ has the property $\left(\beta_{m, p}\right)$ if there exists a neighbourhood $\mathcal{U}$ of $\sigma(A)$ such that for every bounded disc $\mathbf{D}$ containing $\mathcal{U}$, the map $A-z: W^{m}(\mathbf{D}, \mathcal{H}) \rightarrow W^{m}(\mathbf{D}, \mathcal{H})$ satisfies the following: If $\left(f_{n}\right)$ is a sequence in $W^{m}(\mathbf{D}, \mathcal{H})$ such that $\lim _{n \rightarrow \infty}\left\|(A-z) f_{n}\right\|_{W^{m}}=0$ then $\lim _{n \rightarrow \infty}\left\|f_{n}\right\|_{W^{m-p}}=0$.

Let us write $\left(\beta_{m}\right)$ for $\left(\beta_{m, 0}\right)$, and $\left(\beta_{\infty}\right)$ for $\bigcap_{m \in \mathbb{N}}\left(\beta_{m, 0}\right)$.

REMARK 2.5.

(i) $\left(\beta_{m, p}\right) \Rightarrow\left(\beta_{m, q}\right)$ for every $0 \leq q \leq p$.

(ii) $\left(\beta_{m}\right)$ implies that the map in question is bounded below.

(iii) $\left(\beta_{m}\right) \Rightarrow$ SVEP.

(iv) $\left(\beta_{\infty}\right) \Rightarrow\left(\beta_{\epsilon}\right)$.

Proposition 2.6. If $T$ is a hyponormal operator then $T$ has the property $\left(\beta_{m, 2}\right)$ for every integer $m \geq 2$.

Proof. Let $\left(f_{n}\right)_{n} \subset W^{m}(\mathbf{D}, \mathcal{H})$ be such that $\lim _{n \rightarrow \infty}\left\|(T-z) f_{n}\right\|_{W^{m}}=0$. This means that

$$
\lim _{n \rightarrow \infty}\left\|(T-z) \bar{\partial}^{i} f_{n}\right\|_{2, \mathbf{D}}=0, \quad i=0, \ldots, m .
$$

Since $T$ is a hyponormal operator, we have

$$
\lim _{n \rightarrow \infty}\left\|(T-z)^{*} \bar{\partial}^{i} f_{n}\right\|_{2, \mathbf{D}}=0, \quad i=0, \ldots, m .
$$

Using Lemma 2.1, we get

$$
\lim _{n \rightarrow \infty}\left\|(I-P) \bar{\partial}^{i} f_{n}\right\|_{2, \mathbf{D}}=0, \quad i=0, \ldots, m-2 .
$$

This combined with (1) gives

$$
\lim _{n \rightarrow \infty}\left\|(T-z) P \bar{\partial}^{i} f_{n}\right\|_{2, \mathbf{D}}=0, \quad i=0, \ldots, m-2 .
$$

But $T$ has Bishop's property $(\beta)$. So, using Lemma 2.2, we get

$$
\lim _{n \rightarrow \infty}\left\|P \bar{\partial}^{i} f_{n}\right\|_{2, \mathbf{D}}=0
$$

and by (2),

$$
\lim _{n \rightarrow \infty}\left\|\bar{\partial}^{i} f_{n}\right\|_{2, \mathbf{D}}=0, \quad i=0, \ldots, m-2 .
$$

This means $\lim _{n \rightarrow \infty}\left\|f_{n}\right\|_{W^{m-2}}=0$.

In contrast with the SVEP, Bishop's property $(\beta)$ and the property $\left(\beta_{\epsilon}\right)$, we show here that $R S$ and $S R$ do not share exactly the same property $\left(\beta_{k, l}\right)$.

Proposition 2.7. Let $R$ and $S$ be two operators on $\mathcal{H}$. If $R S$ has $\left(\beta_{m, p}\right)$ then $S R$ has $\left(\beta_{m, p+2}\right)$. 
Proof. Let $\left(f_{n}\right)_{n} \subset W^{m}(\mathbf{D}, \mathcal{H})$ be such that

$$
\lim _{n \rightarrow \infty}\left\|(S R-z) f_{n}\right\|_{W^{m}}=0
$$

From the definition of the norm of the Sobolev space, we deduce

$$
\lim _{n \rightarrow \infty}\left\|(S R-z) \bar{\partial}^{i} f_{n}\right\|_{2, \mathbf{D}}=0, \quad i=0, \ldots, m .
$$

By applying $R$, we obtain

$$
\lim _{n \rightarrow \infty}\left\|(R S-z) \bar{\partial}^{i}\left(R f_{n}\right)\right\|_{2, \mathbf{D}}=0, \quad i=0, \ldots, m .
$$

Since $R S$ has the property $\left(\beta_{m, p}\right)$, we get

$$
\lim _{n \rightarrow \infty}\left\|\bar{\partial}^{i}\left(R f_{n}\right)\right\|_{2, \mathbf{D}}=0, \quad i=0, \ldots, m-p .
$$

Applying $S$, we have

$$
\lim _{n \rightarrow \infty}\left\|S R \bar{\partial}^{i} f_{n}\right\|_{2, \mathbf{D}}=0, \quad i=0, \ldots, m-p .
$$

We then deduce from (3) that

$$
\lim _{n \rightarrow \infty}\left\|z \bar{\partial}^{i} f_{n}\right\|_{2, \mathbf{D}}=0, \quad i=0, \ldots, m-p .
$$

Using Lemma 2.1 with $T=0$, we infer

$$
\lim _{n \rightarrow \infty}\left\|(I-P) \bar{\partial}^{i} f_{n}\right\|_{2, \mathbf{D}}=0, \quad i=0, \ldots, m-p-2 .
$$

where $P$ denotes the orthogonal projection of $L^{2}(\mathbf{D}, \mathcal{H})$ onto the Bergman space $A^{2}(\mathbf{D}, \mathcal{H})$.

Then, from (4) and (5), we get

$$
\lim _{n \rightarrow \infty}\left\|z P \bar{\partial}^{i} f_{n}\right\|_{2, \mathbf{D}}=0, \quad i=0, \ldots, m-p-2 .
$$

Since $M_{z}$ is bounded below on the Bergman space, (6) implies that

$$
\lim _{n \rightarrow \infty}\left\|P \bar{\partial}^{i} f_{n}\right\|_{2, \mathbf{D}}=0, \quad i=0, \ldots, m-p-2 .
$$

Then (5) and (7) yield

$$
\lim _{n \rightarrow \infty}\left\|f_{n}\right\|_{W^{m-p-2}}=0
$$

and hence the desired conclusion.

We also have

Proposition 2.8. Let $R$ and $S$ be two operators on $\mathcal{H}$ and let $\mathbf{D}$ be a bounded disc containing $\sigma(R S) \cup\{0\}$. Assume that $R S$ has the property $\left(\beta_{m-1, p}\right)$ for some $m \geq p+2$. Then the map $V: \mathcal{H} \rightarrow H(\mathbf{D})$ defined by

$$
V h=\overline{1 \otimes h}:=1 \otimes h+\overline{(S R-z) W^{m}(\mathbf{D}, \mathcal{H})}
$$

is one-to-one and has closed range, where $1 \otimes h$ denotes the constant function sending any $z$ in $\mathbf{D}$ to $h$, and $H(\mathbf{D}):=W^{m}(\mathbf{D}, \mathcal{H}) \overline{(S R-z) W^{m}(\mathbf{D}, \mathcal{H})}$. 
Proof. Let $\left(h_{n}\right)_{n} \subset \mathcal{H}$ and $\left(f_{n}\right)_{n} \subset W^{m}(\mathbf{D}, \mathcal{H})$ be such that

$$
\lim _{n \rightarrow \infty}\left\|(S R-z) f_{n}+1 \otimes h_{n}\right\|_{W^{m}(\mathbf{D}, \mathcal{H})}=0 .
$$

From the definition of the norm of the Sobolev space, we deduce

$$
\lim _{n \rightarrow \infty}\left\|(S R-z) \bar{\partial}^{i} f_{n}\right\|_{2, \mathbf{D}}=0, \quad i=1, \ldots, m .
$$

By applying $R$, we have

$$
\lim _{n \rightarrow \infty}\left\|(R S-z) \bar{\partial}^{i}\left(R f_{n}\right)\right\|_{2, \mathbf{D}}=0, \quad i=1, \ldots, m,
$$

which means that

$$
\lim _{n \rightarrow \infty}\left\|(R S-z) \bar{\partial}^{1}\left(R f_{n}\right)\right\|_{W^{m-1}}=0 .
$$

Since we have assumed that $R S$ has the property $\left(\beta_{m-1, p}\right)$, we have

$$
\lim _{n \rightarrow \infty}\left\|\bar{\partial}^{1} R f_{n}\right\|_{W^{m-1-p}}=0
$$

or equivalently

$$
\lim _{n \rightarrow \infty}\left\|R \bar{\partial}^{i} f_{n}\right\|_{2, \mathbf{D}}=0, \quad i=1, \ldots, m-p .
$$

Putting this in (9), we get

$$
\lim _{n \rightarrow \infty}\left\|z \bar{\partial}^{i} f_{n}\right\|_{2, \mathbf{D}}=0, \quad i=1, \ldots, m-p .
$$

Now, using Lemma 2.1 with $T=0$ and $i=0$, we obtain (remember that $m \geq p+2$, thus $m-p \geq 2$ )

$$
\left\|(I-P) f_{n}\right\|_{2, \mathbf{D}} \leq C_{\mathbf{D}}\left(\left\|-\bar{z} \bar{\partial}^{1} f_{n}\right\|_{2, \mathbf{D}}+\left\|-\bar{z} \bar{\partial}^{2} f_{n}\right\|_{2, \mathbf{D}}\right) .
$$

From (11) and (10), we have

$$
\lim _{n \rightarrow \infty}\left\|(I-P) f_{n}\right\|_{2, \mathbf{D}}=0,
$$

and this inserted in (8) gives

$$
\lim _{n \rightarrow \infty}\left\|(S R-z) P f_{n}+1 \otimes h_{n}\right\|_{2, \mathbf{D}}=0 .
$$

Now, let $\Gamma$ be a curve in $\mathbf{D}$ surrounding $\sigma(S R) \cup\{0\}$. By using the Hölder inequality, we have

$$
\lim _{n \rightarrow \infty}\left\|\frac{1}{2 \pi i} \int_{\Gamma}\left((S R-z) P f_{n}(z)+1 \otimes h_{n}\right) d \mu(z)\right\|=0 .
$$

By Cauchy's theorem,

$$
\frac{1}{2 \pi i} \int_{\Gamma}(S R-z) P f_{n}(z) d \mu(z)=0,
$$

and so $\lim _{n \rightarrow \infty} h_{n}=0$. We conclude that $V$ is one-to-one and has closed range. 
THEOREM 2.9. Let $R$ and $S$ be two operators on $\mathcal{H}$. If $R S$ has the property $\left(\beta_{m-1, p}\right)$ for some $m \geq p+2$, then $S R$ is subscalar of order $m$.

Proof. Let $V: \mathcal{H} \rightarrow H(\mathbf{D})$ be the operator considered above, defined by $V h=\overline{1 \otimes h}$, where $1 \otimes h$ denotes the constant function $h$. Then $V S R=$ $\bar{M} V$. In particular, $\operatorname{ran} V$ is an invariant subspace of $\bar{M}$. Since $V$ is one-toone and has closed range by Proposition $2.8, S R$ is a subscalar operator of order $m$.

\section{Some applications}

3.1. Aluthge transforms. For a bounded operator $T$ on $\mathcal{H}$, let $U|T|$ be its polar decomposition, where $|T|=\left(T^{*} T\right)^{1 / 2}$ and $U$ is the appropriate partial isometry. The Aluthge transform of $T$ is the operator $\widetilde{T}=|T|^{1 / 2} U|T|^{1 / 2}$ and was first considered by A. Aluthge to extend some inequalities related to hyponormality ([2]).

For $t \in] 0,1[$, the generalized Aluthge transforms of $T$ are the operators $\widetilde{T}(t)=|T|^{1-t} U|T|^{t}$. Note that the Aluthge transform is $\widetilde{T}(1 / 2)$.

Observe that the generalized Aluthge transforms obey the "RS-SR" principle, since if we put $R=|T|^{1-t}$ and $S=U|T|^{t}$, we have $R S=\widetilde{T}(t)$, while $S R=T$. Therefore, we immediately get the following corollary.

Corollary 3.1. Let $T$ be an operator on $\mathcal{H}$. If $\widetilde{T}(t)$ has $\left(\beta_{m, p}\right)$ then $T$ has $\left(\beta_{m, p+2}\right)$.

In particular (when $p=0$ ), we retrieve the following result.

Corollary $3.2([8$, Theorem 1.7]). Let $T$ be an operator on $\mathcal{H}$ and let $\mathbf{D}$ be a bounded disc containing $\sigma(T) \cup\{0\}$. Assume that $\widetilde{T}-z: W^{m}(\mathbf{D}, \mathcal{H}) \rightarrow$ $W^{m}(\mathbf{D}, \mathcal{H})$ is bounded below. If $f_{n}$ is a sequence in $W^{m}(\mathbf{D}, \mathcal{H})$ such that $\lim _{n \rightarrow \infty}\left\|(T-z) f_{n}\right\|_{W^{m}}=0$ then $\lim _{n \rightarrow \infty}\left\|f_{n}\right\|_{W^{m-2}}=0$.

3.2. Backward Aluthge iterates of hyponormal operators. An operator $A \in \mathcal{L}(H)$ is said to be $p$-hyponormal $(p>0)$ if $|A|^{2 p} \geq\left|A^{*}\right|^{2 p}$, where $|A|=$ $\left(A^{*} A\right)^{1 / 2}$. A $1 / 2$-hyponormal operator is called semi-hyponormal ([16]).

The Löwner-Heinz inequality implies that if $A$ is $q$-hyponormal then it is $p$-hyponormal for any $0<p \leq q$. It is known that if $T$ is $p$-hyponormal $(p>0)$, then $\widetilde{T}$ is semi-hyponormal and $\widetilde{\widetilde{T}}$ is hyponormal. $T$ is said to be w-hyponormal ([3]) if $|\widetilde{T}| \geq|T| \geq\left|\widetilde{T}^{*}\right|$, so $\widetilde{T}$ is semi-hyponormal if $T$ is $w$-hyponormal. It is also known $([3])$ that $p$-hyponormal $(p>0)$ operators are $w$-hyponormal.

Consider the iterated Aluthge transforms of $T$ defined by $\widetilde{T}^{(n)}=\widetilde{\widetilde{T}^{(n-1)}}$ for $n \geq 1$ and $\widetilde{T}^{(0)}=T$. 
The following sets were defined in [11]:

$B A I H(k):=\left\{T \in \mathcal{L}(\mathcal{H}): \widetilde{T}^{(k)}\right.$ is a hyponormal operator $\}$.

REMARK 3.3.

(1) $B A I H(0)$ is the set of hyponormal operators.

(2) $B A I H(1):=\{T \in \mathcal{L}(\mathcal{H}): \widetilde{T}$ is a hyponormal operator $\}$ and thus all semi-hyponormal operators are in $B A I H(1)$.

(3) $B A I H(2)$ contains all $w$-hyponormal operators.

We will prove the following result on subscalarity in this class of operators.

Theorem 3.4. If $T \in B A I H(k)$ then:

(1) $T$ has the property $\left(\beta_{m, 2 k+2}\right)$ for every $m \geq 2 k+2$.

(2) $T$ is subscalar of order $2 k+2$.

Proof. As $\widetilde{T}^{(k)}$ is hyponormal, it has the property $\left(\beta_{m, 2}\right)$ for every $m \geq 2$ by Proposition 2.6. Now, by Corollary $3.1, \widetilde{T}^{(k-1)}$ has $\left(\beta_{m, 4}\right)$ for every $m \geq 4$. Repeating the same argument, we find that $\widetilde{T}^{(1)}$ has $\left(\beta_{m, 2 k}\right)$ for every $m \geq 2 k$. Thus $T=\widetilde{T}^{(0)}$ has $\left(\beta_{m, 2 k+2}\right)$ for every $m \geq 2 k+2$ and so it is subscalar of order $2 k+2$ by Theorem 2.9 .

As a corollary, we obtain some well known results of [10] and [14].

Corollary 3.5.

(1) If $T \in B A I H(0)$ (i.e. $T$ is hyponormal) then $T$ is subscalar of order 2 .

(2) If $T$ is w-hyponormal then $T$ is subscalar of order 6 .

Proof. (1) is clear.

(2) Since $T$ is $w$-hyponormal, $T \in B A I H(2)$. Thus, by Theorem 3.4, $T$ is subscalar of order $2 \cdot 2+2=6$.

3.3. p-quasihyponormal operators. We first give the following result which generalizes [12, Lemma 3.2].

Proposition 3.6. Let $T$ be an operator matrix on $\mathcal{M} \oplus \mathcal{M}^{\perp}=\mathcal{H}$ such that $T=\left(\begin{array}{cc}A & C \\ 0 & 0\end{array}\right)$ where $A \in B A I H(k)(\mathcal{M})$ and $\mathcal{M}$ is a closed linear subspace of $\mathcal{H}$. Then $T$ is subscalar of order $4+2 k$.

Proof. Consider

$$
R=\left(\begin{array}{ll}
I & 0 \\
0 & 0
\end{array}\right) \quad \text { and } \quad S=\left(\begin{array}{cc}
A & C \\
0 & I
\end{array}\right) .
$$


Then

$$
S R=\left(\begin{array}{cc}
A & 0 \\
0 & 0
\end{array}\right) \quad \text { and } \quad \widetilde{S} R^{(p)}=\left(\begin{array}{cc}
\widetilde{A}^{p} & 0 \\
0 & 0
\end{array}\right) .
$$

From this we infer that $S R \in B A I H(k)$ if $A \in B A I H(k)$.

By Theorem 3.4, $S R$ has the property $\left(\beta_{m, 2 k+2}\right)$ for every $m \geq 2 k+2$, and by Proposition 2.7 and Theorem $2.9, R S$ has $\left(\beta_{m, 2 k+4}\right)$ if $m \geq 2 k+4$ and $R S=T$ is subscalar of order $2 k+4$.

COROLlary 3.7. If $A$ is a p-hyponormal or a w-hyponormal operator then $T=\left(\begin{array}{cc}A & C \\ 0 & 0\end{array}\right)$ is subscalar of order 8 .

Proof. If $A$ is a $w$-hyponormal operator, then $A$ is in $B A I H(2)$ and we obtain the result from Proposition 3.6.

Now, we recapture the result obtained in [12, Theorem 3.3].

Recall from [15] that an operator $T$ is said to be p-quasihyponormal for $0<p<1$ if $T^{*}\left[\left(T^{*} T\right)^{p}-\left(T T^{*}\right)^{p}\right] T \geq 0$.

COROLlaRY 3.8. Every p-quasihyponormal operator is subscalar of order 6 if $1 / 2 \leq p \leq 1$ and of order 8 if $0<p<1 / 2$.

Proof. It was shown in [12] that a $p$-quasihyponormal operator has the decomposition given in Corollary 3.7, with $A$ a $p$-hyponormal operator.

\section{References}

[1] P. Aiena, Fredholm and Local Spectral Theory with Applications to Multipliers, Kluwer, 2004.

[2] A. Aluthge, On p-hyponormal operators for $0<p<1$, Integral Equations Operator Theory 13 (1990), 307-315.

[3] A. Aluthge and D. Wang, w-hyponormal operators, ibid. 36 (2000), 1-10.

[4] C. Benhida and E. H. Zerouali, Local spectral theory of linear operators $R S$ and $S R$, ibid. 54 (2006), 1-8.

[5] J. B. Conway, Subnormal Operators, Pitman, London, 1981.

[6] J. Eschmeier, Invariant subspaces for subscalar operators, Arch. Math. (Basel) 52 (1989), 562-570.

[7] J. Eschmeier and M. Putinar, Bishop's condition $(\beta)$ and rich extensions of linear operators, Indiana Univ. Math. J. 37 (1988), 325-348.

[8] M.-K. Kim and E. Ko, Some connections between an operator and its Aluthge transform, Glasgow Math. J. 47 (2005), 167-175.

[9] - - - Algebraic extensions of semi-hyponormal operators, Integral Equations Operator Theory 37 (2000), 449-456.

[10] E. Ko, w-hyponormal operators have scalar extensions, ibid. 53 (2005), 336-372.

[11] —, Backward Aluthge iterates of a hyponormal operator have scalar extensions, ibid. 57 (2007), 567-582.

[12] —, p-Quasihyponormal operators have scalar extensions of order 6, J. Math. Anal. Appl. 330 (2007), 80-90. 
[13] K. B. Laursen and M. M. Neumann, Introduction to Local Spectral Theory, Clarendon Press, Oxford, 2000.

[14] M. Putinar, Hyponormal operators are subscalar, J. Operator Theory 12 (1984), 385-395.

[15] K. Tanahashi, A. Uchiyama and M. Chō, Isolated points of spectrum of $(p, k)$-quasihyponormal operators, Linear Algebra Appl. 382 (2004), 221-229.

[16] D. Xia, Spectral Theory of Hyponormal Operators, Operator Theory Adv. Appl. 10, Birkhäuser, Boston, 1983.

UFR de Mathématiques UMR-CNRS 8524

Université de Lille 1

bât. M2

59655 Villeneuve d'Ascq, France

E-mail: benhida@math.univ-lille1.fr
Faculté des Sciences de Rabat BP 1014, Rabat, Maroc E-mail: zerouali@fsr.ac.ma

Received May 27, 2008

Revised version January 27, 2009 